\title{
Continuous Lattices in Formal Topology
}

\author{
Sara Negri \\ Department of Philosophy \\ PL 24, Unioninkatu 40 B \\ 00014 University of Helsinki, Finland \\ e-mail: negri@helsinki.fi
}

\begin{abstract}
A representation of continuous and prime-continuous lattices via formal topology is found. This representation stems from special examples of formal topologies in constructive analysis that give rise to the definition of the classes of locally Stone and locally Scott formal topologies. As an application, a representation theorem for locally compact spaces is obtained.
\end{abstract}

\section{Introduction}

Continuous lattices were first introduced by Dana Scott in $[\mathrm{Sc}]$ as models for untyped $\lambda$-calculus (cf. [Sc1]). They were later extensively studied (cf. [Comp,BH]) and used as a unifying viewpoint for domain theory (as in $[\mathrm{AJ}]$ ).

Continuous lattices also arise in formal topology, in applications to constructive analysis, for instance in the definition of formal reals, formal intervals and formal linear functionals (cf. [NS,CN,CCN]).

The purpose of this paper is to make precise the connection between continuous lattices and formal topology, in such a way that these lattices can be seen as lattices of opens of particular formal spaces.

We start with setting down all the basic notions of formal topology needed. Some definitions differ slightly from the ones given for instance in [S], mainly because we avoid using the positivity predicate. We also single out a relation more general than the cover relation, already introduced in [BS] and called there infinitary preorder, that is needed when facing the problem of representing nondistributive structures.

The basic examples of formal topologies recalled in section 3 all have a cover relation with "nice" approximation features that are summarized in the definition of locally Stone formal topology. The further example of partial reals, i.e., formal reals allowing imprecise points, is analyzed here, and motivates the definition of locally Scott formal topology.

Section 4 is the core of the paper: Here it is shown that these two classes of formal topologies give rise, by considering the corresponding lattices of saturated subsets, to continuous and prime-continuous lattices, respectively, and that every continuous or prime-continuous lattice can be represented in this way via formal topology. The representation for continuous and prime-continuous lattices is part 
of an equivalence that connects infinitary preorders with sup-lattices and formal topologies with frames.

The idea of representing lattices by means of lattices of opens of a topological space goes back to Stone and was often taken over in the literature for various lattice-theoretic structures. By using poinfree spaces instead of topological spaces, we avoid the use of nonconstructive principles. Moreover, by using formal topology, the main results of the spectral theory of continuous lattices (cf. [HL] and [Comp], ch. V) are obtained in a much simpler and more general way.

In the last section we apply our results to get a representation theorem of sober locally compact spaces via formal topology. The axiom of choice is here needed in order to prove extensionality of locally Stone formal topologies. Apart from this unavoidable use of a nonconstructive principle for matching pointfree and point-set topology, all our results are constructive and the proofs elementary.

All the examples of locally Stone formal topologies given here have been implemented in the type-theoretical proof editor HALF (cf. [JC1,JC2]).

\section{Formal Topology}

Formal topologies were introduced by Per Martin-Löf and Giovanni Sambin $([\mathrm{S}, \mathrm{S} 1])$, as a constructive approach to pointfree topology in the tradition of locale theory [I,J,FG], but using Martin-Löf's constructive type theory instead of set theory.

The definition of a formal topology is obtained by abstracting from the definition of a topological space $\langle X, \Omega(X)\rangle$, without mentioning the points. Since a point-set topology can always be presented using one of its bases, the abstract structure that we consider is a commutative monoid $\langle S, \cdot\rangle$ where the set $S$ corresponds to the base of the point-set topology $\Omega(X)$ and the dot corresponds to the operation of intersection between basic subsets.

In a point-set topology any open set is obtained as a union of elements of the base, but union does not make sense if we refuse reference to points; hence we are naturally led to think that an open set may directly correspond to a subset of the set $S$. For this purpose we introduce a relation $\triangleleft$, called cover, between an element $a$ of $S$ and a subset $U$ of $S$. In terms of points, when $b^{*}$ is the set of points of the neighbourhood $b, a \triangleleft U$ can be interpreted as $a^{*} \subseteq \bigcup_{b \in U} b^{*}$. The conditions we require of the cover relation are all justified in such an interpretation in terms of point-set topology.

Definition 1. A formal topology over a set $S$ is a structure

$$
\mathcal{A} \equiv\langle S, \cdot, \triangleleft\rangle
$$

where $\langle S, \cdot\rangle$ is a commutative monoid, $\triangleleft$ is a relation, called cover, between elements and subsets of $S$ such that, for any $a, b \in S$ and $U, V \subseteq S$, the following conditions hold: 


$$
\begin{array}{lc}
\text { reflexivity } & \frac{a \in U}{a \triangleleft U} \\
\text { transitivity } & \frac{a \triangleleft U \quad U \triangleleft V}{a \triangleleft V} \text { where } \quad U \triangleleft V \equiv(\forall u \in U) u \triangleleft V \\
\text { dot-left } & \frac{a \triangleleft U}{a \cdot b \triangleleft U} \\
\text { dot-right } \quad \frac{a \triangleleft U \quad a \triangleleft V}{a \triangleleft U \cdot V} \quad \text { where } \quad U \cdot V \equiv\{u \cdot v \mid u \in U, v \in V\}
\end{array}
$$

An infinitary preorder is a structure $\langle S, \triangleleft\rangle$, where $S$ is a set and $\triangleleft$ is a relation between elements and subsets of $S$ satisfying reflexivity and transitivity.

In contrast to the definition of formal topology given in [S], we do not require the base monoid to have a unit, since the role of the unit element can be taken over by the whole monoid $S$, nor do we have a positivity predicate. The generalization of the definition of formal topology to that of infinitary preorder already appears in $[\mathrm{BS}]$ and will be used here for the representation of nondistributive structures.

In the sequel, we shall omit brackets from singleton sets.

The rule of localization

$$
\frac{a \triangleleft U}{a \cdot b \triangleleft U \cdot b}
$$

is derivable from the rules for a cover, and is actually equivalent to dot-right if the cover satisfies contraction, i.e., for all $a \in S, a \triangleleft a \cdot a$.

Given an infinitary preorder $\mathcal{A}$, we denote with $\operatorname{Sat}(\mathcal{A})$ the collection of saturated subsets of $\mathcal{A}$, that is, of the subsets $U$ of $S$ such that $\mathcal{A} U=U$, where

$$
\mathcal{A} U \equiv\{a \in S \mid a \triangleleft U\} .
$$

We will write $U={ }_{\mathcal{A}} V$ for $U \triangleleft V \& V \triangleleft U$.

$\operatorname{Sat}(\mathcal{A})$ is a complete lattice, with arbitrary joins given by

$$
\bigvee_{i \in I} \mathcal{A} U_{i} \equiv \mathcal{A}\left(\bigcup_{i \in I} U_{i}\right) .
$$

If $\mathcal{A}$ is a formal topology, the meet in $\operatorname{Sat}(\mathcal{A})$ is given by

$$
\mathcal{A} U \wedge \mathcal{A} V \equiv \mathcal{A} U \cap \mathcal{A} V=\mathcal{A}(U \cdot V)
$$

and it is easily seen to be distributive over arbitrary joins, thus making $\operatorname{Sat}(\mathcal{A})$ into a frame (cf. $[\mathrm{S} 1, \mathrm{BS}])$.

In order to connect the pointfree approach to classical point-set topology, a notion of point has to be recovered. Since we reverse the usual conceptual order between points and opens, and take the opens as primitive, points are defined as particular, well behaved, collections of opens. We recall here the definition of formal point of a formal topology: 
Definition 2. Let $\mathcal{A} \equiv\langle S, \cdot \triangleleft\rangle$ be a formal topology. A subset $\alpha$ of $S$ is said to be $a$ formal point if for all $a, b \in S, U \subseteq S$ the following conditions hold:
1. $(\exists a \in S)(a \in \alpha)$;
2. $\frac{a \in \alpha \quad b \in \alpha}{a \cdot b \in \alpha}$;
3. $\frac{a \in \alpha \quad a \triangleleft U}{(\exists b \in U)(b \in \alpha)}$.

We observe that this definition of formal points reduces to the usual one (as given in $[\mathrm{S}]$ ) if the formal topology $\mathcal{A}$ is equipped with a unit 1 and a positivity predicate Pos. Indeed, $1 \in \alpha$ follows from condition 1 and $a \triangleleft 1$ for all $a$; the rule $\frac{a \in \alpha}{\operatorname{Pos}(a)}$ follows from the rule of positivity, in the form $a \triangleleft a^{+}$, where $U^{+} \equiv\{b \in U \mid \operatorname{Pos}(b)\}$, and 3 .

In order to maintain the usual intuition on points, we will write $\alpha \Vdash a$ ( $\alpha$ forces $a$, or $\alpha$ is a point in $a$ ) in place of $a \in \alpha$.

Formal topologies can be made into a category, FTop, whose objects are formal topologies and whose morphisms, corresponding to the inverses of continuous functions between topological spaces, are defined as follows (cf. the appendix of $[\mathrm{NV}]$ for a more detailed motivation of this definition):

Definition 3. Let $\mathcal{A}=\left\langle S, \cdot_{\mathcal{A}}, \triangleleft_{\mathcal{A}}\right\rangle$, and $\mathcal{B}=\left\langle T, \cdot_{\mathcal{B}}, \triangleleft_{\mathcal{B}}\right\rangle$ be two formal topologies. A morphism from $\mathcal{A}$ to $\mathcal{B}$ is a map $f$, from elements of $S$ to subsets of $T$, such that the following conditions are satisfied:

$$
\begin{aligned}
& \text { 1. } f(S)={ }_{\mathcal{B}} T ; \\
& \text { 2. } f(a \cdot \mathcal{A} b)={ }_{\mathcal{B}} f(a) \cdot \mathcal{B} f(b) ; \\
& \text { 3. } \frac{a \triangleleft_{\mathcal{A}} U}{f(a) \triangleleft_{\mathcal{B}} \bigcup_{b \in U} f(b)} \text {. }
\end{aligned}
$$

Two morphisms $f$ and $g$ from $\mathcal{A}$ to $\mathcal{B}$ are equal if, for all $a \in S, f(a)={ }_{\mathcal{B}} g(a)$. If $\mathcal{A}$ and $\mathcal{B}$ are infinitary preorders, a morphism from $\mathcal{A}$ to $\mathcal{B}$ is a map $f$ from $S$ to $\mathcal{P}(T)$ such that the third of the above conditions is satisfied.

In the presence of the unit, the first condition above is equivalent to the usual one,

$$
f\left(1_{\mathcal{A}}\right)={ }_{\mathcal{B}} 1_{\mathcal{B}},
$$

and ensures that the corresponding frame morphism (defined below) preserves finite meets, hence, in particular, the top element, that can be given as the meet of the empty set.

We can extend $S a t$ from objects to morphisms as follows: For $U \in \operatorname{Sat}(\mathcal{A})$ and $f$ a morphism from $\mathcal{A}$ to $\mathcal{B}$, we put

$$
\operatorname{Sat}(f)(U) \equiv \mathcal{B}\left(\bigcup_{b \in U} f(b)\right) .
$$

In this way we obtain a functor that gives an equivalence between the category of infinitary preorders and the category of sup-lattices (cf. [JT] for the latter). 
Such an equivalence restricts to an equivalence between the category of formal topologies and the category of frames.

We recall that for any formal topology $\mathcal{A}$, the formal space $\operatorname{Pt}(\mathcal{A})$ of formal points on $\mathcal{A}$ can be endowed with a topology, called the extensional topology. A base of this topology is given by the family $\{\operatorname{ext}(a)\}_{a \in S}$ where $a \in S$ and $\operatorname{ext}(a)$ is the collection of formal points forcing $a$.

By the condition of monononicity for formal points, if $a \triangleleft U$, then for any formal point $\alpha$ such that $\alpha \Vdash a$, we have $\alpha \Vdash U$. The converse does not necessarily hold, and indeed is the defining property of extensional formal topologies.

The following result is the counterpart of a well known result for spatial locales; its proof can be easily obtained from the proof of the latter (cf. $[\mathrm{J}]$ ):

Theorem 1. The category of extensional formal topologies is equivalent to the category of sober topological spaces with continuous maps.

In section 5 we will specialize this result to an equivalence between a particular class of formal topologies and locally compact sober topological spaces.

We recall from [S] that a cover $\triangleleft$ on a base $S$ is called a Stone cover if, for all $a \in S$ and $U \subseteq S, a \triangleleft U$ implies $a \triangleleft U_{0}$ for some finite subset $U_{0}$ of $U$ (written $\left.U_{0} \subseteq_{\omega} U\right)$. We remark that the notion of being Stone can apply not just to a cover, but, with more generality, to any infinitary preorder. In particular, we have:

Proposition 1. If $\mathcal{A}$ is a Stone infinitary preorder, then $\operatorname{Sat}(\mathcal{A})$ is an algebraic lattice, i.e., it has a basis of compact elements.

Proof: The complete lattice $\operatorname{Sat}(\mathcal{A})$ is generated by compact elements since, for any element $U$ we have $U=\bigvee_{a \in U} \mathcal{A} a$, and every element of the base in compact by definition of Stone infinitary preorder.

We recall from $[\mathrm{N}]$ that we also have the related result (seen now as a corollary of the above one just by adding distributivity):

Proposition 2. If $\mathcal{A}$ is a Stone formal topology, then $\operatorname{Sat}(\mathcal{A})$ is a coherent frame.

We say that a cover $\triangleleft$ on $S$ is a $S$ cott cover (or alternatively, as in [Si], that it has the Scott property) if for all $a \in S$ and $U \subseteq S$,

$$
a \triangleleft U \text { implies } a \triangleleft b \text { for some } b \in U
$$

We remark that this notion of Scott cover differs from the one given in [S] and used in [SVV], where the condition (1) is only required for positive elements of the base.

As above, this notion of being Scott, can apply as well to an infinitary preorder.

Given two covers $\triangleleft_{1}$ and $\triangleleft_{2}$ on the same base $S$, we say that $\triangleleft_{2}$ is a quotient of $\triangleleft_{1}$ (or is greater than $\triangleleft_{1}$ ) if for all $a \in S$ and $U \subseteq S$,

$$
a \triangleleft_{1} U \Rightarrow a \triangleleft_{2} U .
$$


The Stone (resp. Scott) compactification of a cover is defined as the greatest Stone (resp. Scott) cover of which the given cover is a quotient. They are defined, respectively, by

$$
\begin{aligned}
& a \triangleleft_{f} U \equiv\left(\exists U_{0} \subseteq_{\omega} U\right)\left(a \triangleleft U_{0}\right) \\
& a \triangleleft_{s} U \equiv(\exists b \in U)(a \triangleleft b) .
\end{aligned}
$$

The Stone and Scott compactifications for infinitary preorders are defined in the same way.

\section{Real numbers, intervals and linear functionals}

In this section we show examples of formal topologies in which the cover is presented via a Stone or Scott cover. They are all given by means of a finitary inductive definition (cf. $[\mathrm{A}]$ ), where each rule involved has only finitely many premises. We start with the topology of formal reals (cf. [N1], [NS], [CN], [CCN]). This is our motivating example and we therefore recall the presentation in detail.

The following definition, which is the one used in [JC2], was proposed by Thierry Coquand.

Definition 4. The formal topology of formal reals is the structure

$$
\mathcal{R} \equiv\left\langle Q \times Q, \cdot, \triangleleft_{\mathcal{R}}\right\rangle,
$$

where $Q$ is the set of rational numbers and the monoid operation is defined by $(p, q) \cdot(r, s) \equiv(\max (p, r), \min (q, s)) ;$ the cover $\triangleleft_{\mathcal{R}}$ is defined by

$$
(p, q) \triangleleft_{\mathcal{R}} U \equiv\left(\forall p^{\prime}, q^{\prime}\right)\left(p<p^{\prime}<q^{\prime}<q \rightarrow\left(p^{\prime}, q^{\prime}\right) \triangleleft_{\mathcal{R}_{f}} U\right),
$$

where the relation $\triangleleft_{\mathcal{R}_{f}}$ is inductively defined by

$$
\begin{aligned}
& \text { 1. } \frac{q \leq p}{(p, q) \triangleleft_{\mathcal{R}_{f}} U} ; \\
& \text { 2. } \frac{(p, q) \in U}{(p, q) \triangleleft_{\mathcal{R}_{f}} U} ; \\
& \text { 3. } \frac{(p, s) \triangleleft_{\mathcal{R}_{f}} U \quad(r, q) \triangleleft_{\mathcal{R}_{f}} U \quad p \leq r<s \leq q}{(p, q) \triangleleft_{\mathcal{R}_{f}} U} ; \\
& \text { 4. } \frac{\left(p^{\prime}, q^{\prime}\right) \triangleleft_{\mathcal{R}_{f}} U \quad p^{\prime} \leq p<q \leq q^{\prime}}{(p, q) \triangleleft_{\mathcal{R}_{f}} U} .
\end{aligned}
$$

In [CN], it is proved that both $\triangleleft_{\mathcal{R}}$ and $\triangleleft_{\mathcal{R}_{f}}$ are covers, the latter being the Stone compactification of the former.

The proof that $\triangleleft_{\mathcal{R}}$ is a cover makes essential use of a lemma, that we recall here from $[\mathrm{CN}]$ since it will be used elsewhere in this paper and later presented in a more abstract way:

Lemma 1. Suppose $(p, q) \triangleleft_{\mathcal{R}_{f}} U, U \triangleleft_{\mathcal{R}} V$, and let $p<p^{\prime}<q^{\prime}<q$. Then $\left(p^{\prime}, q^{\prime}\right) \triangleleft_{\mathcal{R}_{f}} V$. 
We can widen the collection of formal reals by allowing also "imprecise" numbers. The presentation of the formal topology having partial reals as formal points is obtained by omitting the first and third axiom from the definition we gave for $\triangleleft_{\mathcal{R}_{f}}$ :

Definition 5. The formal topology of partial reals is the structure

$$
\mathcal{P} \mathcal{R} \equiv\langle Q \times Q, \cdot, \triangleleft \mathcal{P} \mathcal{R}\rangle,
$$

with $\triangleleft_{\mathcal{P} \mathcal{R}}$ defined as in definition 4 from a relation $\triangleleft_{\mathcal{P} \mathcal{R}_{s}}$ fulfilling conditions 2 and 4 of the definition of $\triangleleft_{\mathcal{R}_{f}}$.

It is easy to prove the following:

Proposition 3. 1. The relations $\triangleleft_{\mathcal{P R}}$ and $\triangleleft_{\mathcal{P} \mathcal{R}_{s}}$ are covers.

2. For all $a \in Q \times Q$ and $U \subseteq Q \times Q, a \triangleleft_{\mathcal{P} \mathcal{R}_{s}} U$ implies $a \triangleleft_{\mathcal{P R}} U$.

3. For all $a \in Q \times Q$ and $U \subseteq Q \times Q, a \triangleleft \mathcal{P R}_{s} U$ implies that there exists $b \in U$ such that $a \triangleleft \mathcal{P \mathcal { R }}_{s} b$.

We will denote with $\operatorname{Pt}(\mathcal{P} \mathcal{R})$ the formal points of $\mathcal{P} \mathcal{R}$.

Formal reals can be described as well-located partial reals. First, we recall the following (cf. [NS]):

Definition 6. For any rational $p, \bar{p}$ is the formal real $\{(r, s): r<p<s\}$.

Proposition 4. If $\alpha$ and $\beta$ are formal reals, the relations given by

$$
\begin{aligned}
& \alpha<\beta \equiv(\exists(p, q) \in \alpha)(\exists(r, s) \in \beta)(q<r) ; \\
& \alpha \leq \beta \equiv \neg(\beta<\alpha) ; \\
& \alpha \# \beta \equiv \alpha<\beta \vee \beta<\alpha .
\end{aligned}
$$

are relations of strict linear order, partial order and apartness, respectively.

Then we can easily characterize those partial reals that are also formal reals:

Proposition 5. Let $\alpha \in P t(\mathcal{P R})$. Then the following are equivalent:

1. $\alpha \in \operatorname{Pt}(\mathcal{R})$.

2. $(\forall p, q)(p<q \rightarrow \bar{p}<\alpha \vee \alpha<\bar{q})$.

3. $\left(\forall k \in Q^{+}\right)(\exists(p, q))(q-p<k \& \alpha \Vdash(p, q))$.

A global version of the above characterization is given by the following:

Corollary 1. $\operatorname{Pt}(\mathcal{P R})=\operatorname{Pt}(\mathcal{R})$ iff the relation \# is an apartness relation on $\operatorname{Pt}(\mathcal{P R})$.

We now turn to another example of inductively generated formal topology.

Similarly to the definition of formal reals, we can define the formal space $[a, b]$ that corresponds to the closed interval of the real line with rational endpoints $a$ and $b(\mathrm{cf}$. [CN]): The base is the same as the base of the space of formal reals, and the cover relation is defined by

$$
(p, q) \triangleleft_{[a, b]} U \equiv(p, q) \triangleleft_{\mathcal{R}} U \cup C([a, b])
$$


where $C([a, b])=\{(r, a) \mid r<a\} \cup\{(b, s) \mid b<s\}$. It is then proved that the formal points of this space are exactly the formal reals $\alpha$ with $a \leq \alpha \leq b$.

There is an alternative definition of this space in which an explicit presentation of its Stone compactification is given. This is achieved by adding to the axioms for the finitary cover $\triangleleft_{\mathcal{R}_{f}}$ of formal reals two axioms expressing the fact that intervals not overlapping with $[a, b]$ are covered by anything.

Definition 7. Let $a, b$ be rationals with $a<b$. The formal topology of the closed interval $[a, b]$ is the structure

$$
[a, b] \equiv\left\langle Q \times Q, \cdot, \triangleleft^{\prime}\right\rangle,
$$

with the relation $\triangleleft^{\prime}$ defined by

$$
(p, q) \triangleleft^{\prime} U \equiv\left(\forall p^{\prime}, q^{\prime}\right)\left(p<p^{\prime}<q^{\prime}<q \rightarrow\left(p^{\prime}, q^{\prime}\right) \triangleleft_{f}^{\prime} U\right),
$$

and the relation $\triangleleft_{f}^{\prime}$ is inductively defined by

$$
\begin{aligned}
& \text { 1. } \frac{(p, q) \triangleleft_{\mathcal{R}_{f}} U}{(p, q) \triangleleft_{f}^{\prime} U} ; \\
& \text { 2. } \frac{q \leq a}{(p, q) \triangleleft_{f}^{\prime} U} ; \\
& \text { 3. } \frac{b \leq p}{(p, q) \triangleleft_{f}^{\prime} U} \text {. }
\end{aligned}
$$

Then we have

Proposition 6. The relation $\triangleleft^{\prime}$ is a cover, equivalent to the cover $\triangleleft_{[a, b]}$, with Stone compactification given by $\triangleleft_{f}^{\prime}$.

Proof: We start with proving that $\triangleleft_{f}^{\prime}$ is a cover: Since we already know that $\triangleleft_{\mathcal{R}_{f}}$ is a cover, to get to the conclusion we only need to prove that transitivity, dot - left and localization hold when $(p, q) \triangleleft_{f}^{\prime} U$ is derived from the new axioms 2 and 3 in definition 7 , which is straightforward. In order to prove that $\triangleleft^{\prime}$ is a cover, we observe that lemma 1 also holds when $\triangleleft_{\mathcal{R}}$ and $\triangleleft_{\mathcal{R}_{f}}$ are replaced with $\triangleleft^{\prime}$ and $\triangleleft_{f}^{\prime}$, respectively: this is seen by the trivial inspection of the cases in which $(p, q) \triangleleft_{f}^{\prime} U$ is obtained by axiom 2 or 3 . This directly implies that $\triangleleft^{\prime}$ satisfies transitivity. The verification that it satisfies reflexivity, dot-left and dot-right is easy.

We proceed with proving the equivalence between $\triangleleft^{\prime}$ and $\triangleleft_{[a, b]}$. Suppose that $(p, q) \triangleleft^{\prime} U$ and let $p<p^{\prime}<q^{\prime}<q$. Then by definition we have $\left(p^{\prime}, q^{\prime}\right) \triangleleft_{f}^{\prime} U$. We then prove by induction on the derivation of $\left(p^{\prime}, q^{\prime}\right) \triangleleft_{f}^{\prime} U$ that $\left(p^{\prime}, q^{\prime}\right) \triangleleft_{\mathcal{R}_{f}} U \cup$ $C([a, b])$ follows. If it is obtained by axiom 1 the claim is trivial. If it is derived by 2 or 3 , then $\left(p^{\prime}, q^{\prime}\right) \triangleleft_{\mathcal{R}_{f}} C([a, b])$ and therefore the conclusion follows by reflexivity and transitivity for $\triangleleft_{\mathcal{R}_{f}}$. We have thus proved that $(p, q) \triangleleft_{\mathcal{R}} U \cup C([a, b])$. Conversely, assume that $(p, q) \triangleleft_{\mathcal{R}} U \cup C([a, b])$, and let $p<p^{\prime}<q^{\prime}<q$. Then we have $\left(p^{\prime}, q^{\prime}\right) \triangleleft_{\mathcal{R}_{f}} U \cup C([a, b])$. We then argue by induction on this derivation in order to prove that $\left(p^{\prime}, q^{\prime}\right) \triangleleft_{f}^{\prime} U$. For axiom 1 the claim is trivial. Then suppose 
that $\left(p^{\prime}, q^{\prime}\right) \in U \cup C([a, b])$. If $\left(p^{\prime}, q^{\prime}\right) \in U$, the conclusion follows by reflexivity and transitivity for $\triangleleft_{f}^{\prime}$. If $\left(p^{\prime}, q^{\prime}\right) \in C([a, b])$, then by axioms 2 and 3 we get $\left(p^{\prime}, q^{\prime}\right) \triangleleft_{f}^{\prime} U$. For axioms 3 and 4 we apply the inductive hypotheses to the premises.

The proof that $\triangleleft_{f}^{\prime}$ is a Stone cover is done by induction on the derivation: If $(p, q) \triangleleft_{f}^{\prime} U$ is obtained from $(p, q) \triangleleft_{\mathcal{R}_{f}} U$ then the information that $\triangleleft_{\mathcal{R}_{f}}$ is a Stone cover provides us with a finite subcover. If it is obtained from $q \leq a$ or $b \leq p$ then the empty set is a finite subcover of $(p, q)$.

The verification that $\triangleleft^{\prime}$ is a quotient of $\triangleleft_{f}^{\prime}$ is straightforward.

Finally, we have to prove that if $(p, q) \triangleleft^{\prime} U$, with $U$ a finite subset of $Q \times Q$, then $(p, q) \triangleleft_{f}^{\prime} U$. By the above proof of equivalence of the two covers, $(p, q) \triangleleft^{\prime} U$ implies $(p, q) \triangleleft_{[a, b]} U$, that is, $(p, q) \triangleleft_{\mathcal{R}} U \cup C([a, b])$. By localizing to $(p, q)$ we get $(p, q) \triangleleft_{\mathcal{R}} U \cup\{(p, a),(b, q)\}$, and therefore, since $\triangleleft_{\mathcal{R}_{f}}$ is the Stone compactification of $\triangleleft_{\mathcal{R}}$, we get $(p, q) \triangleleft_{\mathcal{R}_{f}} U \cup\{(p, a),(b, q)\}$. By induction on the derivation of $(p, q) \triangleleft_{\mathcal{R}_{f}} U \cup\{(p, a),(b, q)\}$ (the inductive hypothesis being that $(p, q) \triangleleft_{\mathcal{R}_{f}} U \cup$ $\{(x, a),(b, y)\}$ implies $(p, q) \triangleleft_{f}^{\prime} U$, for arbitrary $x$ and $\left.y\right)$, we find that $(p, q) \triangleleft_{f}^{\prime} U$.

We conclude this section by recalling another example of inductively generated formal topology, the topology of linear and continuous functionals of norm $\leq 1$ from a seminormed linear space to the reals (cf. $[\mathrm{CCN}])$.

Seminormed spaces can be defined as in $[\mathrm{MP}]$ :

Definition 8. A seminormed space $A$ on the rationals $Q$ is a linear space $A$ on $Q$ together with a mapping

$$
N: Q^{+} \longrightarrow \mathcal{P}(A)
$$

from the positive rationals to the subsets of $A$ satisfying the following conditions for $x, x^{\prime} \in A, q, q^{\prime} \in Q^{+}$:

$$
\begin{aligned}
& \text { N1. } x \in N(q) \rightarrow\left(\exists q^{\prime}<q\right)\left(x \in N\left(q^{\prime}\right)\right) ; \\
& \text { N2. } \exists q)(x \in N(q)) ; \\
& \text { N3. } x \in N(q) \& x^{\prime} \in N\left(q^{\prime}\right) \rightarrow x+x^{\prime} \in N\left(q+q^{\prime}\right) ; \\
& \text { N4. } x \in N\left(q^{\prime}\right) \rightarrow q x \in N\left(q q^{\prime}\right) ; \\
& \text { N5. } x \in N(q) \rightarrow-x \in N(q) ; \\
& \text { N6. } 0 \in N(q) \text {. }
\end{aligned}
$$

The basic opens of the formal space $\mathcal{L}(A)$ of linear functionals of norm $\leq 1$ are finite sets of the form

$$
w \equiv\left\{\left\langle x_{1} \in I_{1}\right\rangle, \ldots,\left\langle x_{n} \in I_{n}\right\rangle\right\},
$$

where $x_{1}, \ldots, x_{n}$ are elements of $A$ and $I_{1}, \ldots, I_{n}$ are rational intervals. The intuitive reading of a basic open is that of a neighbourhood of functionals in the weak topology. We use the notation $\left\langle x_{1} \in I_{1}, \ldots, x_{n} \in I_{n}\right\rangle$ for $\left\{\left\langle x_{1} \in I_{1}\right\rangle, \ldots,\left\langle x_{n} \in\right.\right.$ $\left.\left.I_{n}\right\rangle\right\}$. 
We obtain with the operation

$$
w_{1} w_{2} \equiv w_{1} \cup w_{2}
$$

a commutative and idempotent monoid with unit given by the empty set. We will denote with $S_{\mathcal{L}(A)}$ such a base of $\mathcal{L}(A)$.

With $I=(p, q)$ and $J=(r, s)$, we write $I<J$ (resp. $I \leq J)$ for $r<p<q<s$ (resp. $r \leq p<q \leq s$ ), and $t I$ for $(t p, t q)$ when $t \geq 0$ and for $(t q, t p)$ when $t<0$.

Let $w \equiv\left\langle x_{1} \in I_{1}, \ldots, x_{n} \in I_{n}\right\rangle$, then define

$$
\begin{aligned}
w \leq\langle x \in I\rangle \equiv & \left(\exists\left\langle x_{i_{1}} \in I_{i_{1}}\right\rangle, \ldots,\left\langle x_{i_{p}} \in I_{i_{p}}\right\rangle \in w\right) \\
& \left(x_{i_{1}}=\ldots=x_{i_{p}}=x \& I_{i_{1}} \cdot \ldots \cdot I_{i_{p}} \leq I\right)
\end{aligned}
$$

and

$$
w \leq w^{\prime} \equiv\left(\forall\langle x \in I\rangle \in w^{\prime}\right)(w \leq\langle x \in I\rangle) .
$$

Then, without assuming decidability of equality in $A, \leq$ is a reflexive and transitive relation.

Equality between basic neighbourhoods is subset equality

$$
w=w^{\prime} \equiv(\forall\langle x \in I\rangle)\left(\langle x \in I\rangle \in w \Leftrightarrow\langle x \in I\rangle \in w^{\prime}\right) .
$$

The relation $\triangleleft_{f}$ is inductively defined by:

$C 1 \frac{w \in U}{w \triangleleft_{f} U} ;$

$C 2 \frac{w \leq w^{\prime} w^{\prime} \triangleleft_{f} U}{w \triangleleft_{f} U} ;$

$C 3 \frac{V \text { finite } \quad I \triangleleft_{\mathcal{R}_{f}} V \quad(\forall J \in V)\left(\langle x \in J\rangle w^{\prime} \triangleleft_{f} U\right)}{\langle x \in I\rangle w^{\prime} \triangleleft_{f} U} ;$

$C_{4} \frac{\langle x+y \in I+J\rangle w^{\prime} \triangleleft_{f} U}{\langle x \in I, y \in J\rangle w^{\prime} \triangleleft_{f} U} ;$

$C 5 \frac{r \neq 0 \quad\langle r x \in r I\rangle w^{\prime} \triangleleft_{f} U}{\langle x \in I\rangle w^{\prime} \triangleleft_{f} U} ;$

$C 6 \frac{x \in N(1) \quad\langle x \in(-1,1)\rangle w \triangleleft_{f} U}{w \triangleleft_{f} U}$.

Then $\triangleleft$ is defined by

$\left\langle x_{1} \in I_{1}, \ldots, x_{n} \in I_{n}\right\rangle \triangleleft U \equiv\left(\forall J_{1}<I_{1}, \ldots, J_{n}<I_{n}\right)\left(\left\langle x_{1} \in J_{1}, \ldots, x_{n} \in J_{n}\right\rangle \triangleleft_{f} U\right)$.

In $[\mathrm{CCN}]$ it is then proved that $\triangleleft$ is a cover and that it is a quotient of the Stone cover $\triangleleft_{f}$. 


\section{Continuous and prime-continuous lattices in formal topology}

The examples of the topologies of formal reals, intervals and linear functionals seen in the previous section motivate us to introduce the definition of two particular classes of formal topologies. We will see that these formal topologies permit a representation theorem for continuous and prime-continuous lattices.

Definition 9. A formal topology $\mathcal{A} \equiv\langle S, \cdot, \triangleleft\rangle$ is called locally Stone if there exists a map $i$ from elements to subsets of $S$ such that, for all $a \in S$ and $U \subseteq S$,

$$
a \triangleleft U \Leftrightarrow(\forall b \in i(a))\left(b \triangleleft_{f} U\right)
$$

where $\triangleleft_{f}$ is a Stone cover of which $\triangleleft i$ is a quotient.

A formal topology is called locally Scott if there exists a map $i$ as above such that, for all $a \in S$ and $U \subseteq S$,

$$
a \triangleleft U \Leftrightarrow(\forall b \in i(a))\left(b \triangleleft_{s} U\right)
$$

where $\triangleleft_{s}$ is a Scott cover of which $\triangleleft$ is a quotient.

It is clear from the results in the previous section that the topology of formal reals and of formal closed intervals are locally Stone and the topology of partial reals is locally Scott. In these examples we have

$$
i((p, q))=\left\{\left(p^{\prime}, q^{\prime}\right): p<p^{\prime}<q^{\prime}<q\right\} .
$$

The topology of linear functionals is locally Stone, and we have

$$
i\left(\left\langle x_{1} \in I_{1}, \ldots, x_{n} \in I_{n}\right\rangle\right)=\left\{\left\langle x_{1} \in J_{1}, \ldots, x_{n} \in J_{n}\right\rangle \mid J_{1}<I_{1}, \ldots, J_{n}<I_{n}\right\} .
$$

The definitions of locally Stone and locally Scott infinitary preorders are obtained from the above in the obvious way, simply by replacing "formal topology" with "infinitary preorder".

We observe that a Stone (resp. Scott) formal topology $\mathcal{A} \equiv\langle S, \cdot, \triangleleft\rangle$ is locally Stone (resp. Scott) with $i(a)=\{a\}$ and $\triangleleft_{f}\left(\right.$ resp. $\left.\triangleleft_{s}\right)$ the cover $\triangleleft$ itself. The same holds for infinitary preorders.

We have, with the notation introduced above:

Proposition 7. If $\mathcal{A}$ is a locally Stone formal topology (or infinitary preorder), then for all a in the base $S$,

$$
a={ }_{\mathcal{A}} i(a) .
$$

Proof: For all $b \in i(a)$ we have, by reflexivity, $b \triangleleft_{f} i(a)$, so by definition of locally Stone cover (or infinitary preorder) we have $a \triangleleft i(a)$.

Conversely, from $a \triangleleft a$, we have that for all $b \in i(a), b \triangleleft_{f} a$. Since $\triangleleft$ is a quotient of $\triangleleft_{f}$, we also have that for all $b \in i(a), b \triangleleft a$, i.e., $i(a) \triangleleft a$.

We proceed with recalling some definitions and basic facts from domain theory (see [Comp], [AJ]). 
Definition 10. Let $L$ be a complete lattice and let $x, y \in L$. We say that $x$ approximates $y$, or $x$ is way-below $y$, and write

$$
x \ll y,
$$

if, for all directed subsets $A$ of $L, y \leq \bigvee A$ implies that there exists a $\in A$ such that $x \leq a$.

We say that $x$ prime-approximates $y$, and write

$$
x \lll y,
$$

if, for all subsets $A$ of $L, y \leq \bigvee A$ implies that there exists $a \in A$ such that $x \leq a$.

A complete lattice is continuous if, for all $x \in L, x=\bigvee\{y \mid y \ll x\}$.

A complete lattice is prime-continuous if, for all $x \in L, x=\bigvee\{y \mid y \lll x\}$.

Definition 11. A subset $S$ of a continuous lattice $L$ is a base of $L$ if, for all $a \in L$, the subset $\downarrow_{S} a \equiv\{x \in S \mid x \ll a\}$ is directed with supremum $a$.

A subset $S$ of a prime-continuous lattice $L$ is a base of $L$ if, for all $a \in L$, the subset $\Downarrow_{S} a \equiv\{x \in S \mid x \lll a\}$ is directed with supremum $a$.

Proposition 8 ((Interpolation property)). In a continuous lattice, if $a \ll b$, there exists $c$ such that $a \ll c \ll b$. In a prime-continuous lattice, if $a \lll b$, there exists $c$ such that $a \lll c \lll b$.

Proof: Cf. [J], VII 2.4, and [AJ], p. 69.

We observe that given $a \ll b$ in a continuous lattice with base $S$, the interpolating $c$ is not necessarily in $S$, but we can find $c_{1}, \ldots, c_{n}$ in $S$ such that $a \ll \bigvee_{i=1}^{n} c_{i} \ll b$ (for a proof cf. [Si]).

The following two results are instances of theorems 7.1.1 and 7.1.3 in [AJ] (for the equational characterization of the class of continuous lattices, see also theorem I, 2.3 in [Comp]). The proofs of these stronger statements require the axiom of choice, whereas those we need have entirely constructive proofs:

Lemma 2. A continuous lattice $L$ satisfies the directed infinite distributive law: For any $a \in L$ and any directed subset $\left\{b_{i}: i \in I\right\}$ of $L$,

$$
a \wedge \bigvee_{i \in I} b_{i}=\bigvee_{i \in I} a \wedge b_{i}
$$

Proof: The inequality from right to left holds in any lattice, so the claim amounts to proving that $a \wedge \bigvee_{i \in I} b_{i} \leq \bigvee_{i \in I} a \wedge b_{i}$. Let $x \ll a \wedge \bigvee_{i \in I} b_{i}$. Then $x \ll \bigvee_{i \in I} b_{i}$ and therefore there exists $i \in I$ such that $x \leq b_{i}$ and indeed such that $x \leq a \wedge b_{i}$ since $x \leq a$. Thus $x \leq \bigvee_{i \in I} a \wedge b_{i}$. Since $L$ is continuous this proves the claim.

Lemma 3. A distributive continuous lattice $L$ satisfies the infinite distributive law: For any $a \in L$ and any subset $\left\{b_{i}: i \in I\right\}$ of $L$

$$
a \wedge \bigvee_{i \in I} b_{i}=\bigvee_{i \in I} a \wedge b_{i}
$$


Proof: Just observe that $\bigvee_{i \in I} b_{i}=\bigvee_{I_{0} \subseteq_{\omega} I}\left\{\bigvee_{i \in I_{0}} b_{i}\right\}$ and that the set of finite subsets of $I$ is directed and so are the suprema indexed on these.

The above lemma explains why distributive continuous lattices are also called continuous Heyting algebras.

Lemma 4. A prime-continuous lattice $L$ satisfies the infinite distributive law.

Proof: As 2, using prime-continuity instead of continuity.

We proceed by showing that examples of continuous and prime-continuous lattices are given by the frames of saturated subsets of locally Stone and locally Scott infinitary preorders.

Theorem 2. If $\mathcal{A}$ is a locally Stone infinitary preorder, then $\operatorname{Sat}(\mathcal{A})$ is a continuous lattice.

Proof: Let $U \in \operatorname{Sat}(\mathcal{A})$. We have to prove that $U=\bigvee\{V \in \operatorname{Sat}(\mathcal{A}): V \ll U\}$, i.e., $U=\mathcal{A}(\bigcup\{V \in \operatorname{Sat}(\mathcal{A}): V \ll U\})$, or equivalently, since $V \ll U$ in $\operatorname{Sat}(\mathcal{A})$ implies $V \triangleleft U, U \triangleleft \bigcup\{V \in \operatorname{Sat}(\mathcal{A}): V \ll U\}$. Observe that $b \in i(a)$ implies $\mathcal{A} b \ll \mathcal{A} a$ : If $\mathcal{A} a \leq \bigvee_{i \in I} U_{i}$ where $\left\{U_{i}: i \in I\right\}$ is a directed subset of $\operatorname{Sat}(\mathcal{A})$, then $a \triangleleft \bigcup_{i \in I} U_{i}$; since $b \in i(a), b \triangleleft_{f} \bigcup_{i \in I} U_{i}$, and since the family is directed and $\triangleleft_{f}$ is a Stone infinitary preorder, there exists $i \in I$ such that $b \triangleleft_{f} U_{i}$, hence such that $b \triangleleft U_{i}$, so $\mathcal{A} b \leq \mathcal{A} U_{i}$. For all $a \in U$ and for all $b \in i(a)$, we have $\mathcal{A} b \ll \mathcal{A} a, \mathcal{A} a \leq U$ and therefore $\mathcal{A} b \ll U$, thus, since $U \triangleleft \bigcup_{a \in U}\{b: b \in i(a)\}$, we have the claim.

Corollary 2. If $\mathcal{A}$ is a locally Stone formal topology, then $\operatorname{Sat}(\mathcal{A})$ is a distributive continuous lattice.

We also obtain the converses of the above results, namely:

Theorem 3. Every continuous lattice is isomorphic to the lattice of saturated subsets of a locally Stone infinitary preorder. If it is distributive it is isomorphic to the lattice of saturated subsets of a locally Stone formal topology.

Before proving this theorem we need a couple of lemmas:

Lemma 5. Let $L$ be a continuous lattice. If $b \ll u_{1} \vee \ldots \vee u_{n}$, there exist $b_{1} \ll u_{1}$, $\ldots, b_{n} \ll u_{n}$ such that $b \leq b_{1} \vee \ldots \vee b_{n}$.

Proof: By continuity we have

$$
u_{1} \vee \ldots \vee u_{n}=\bigvee_{b_{1} \ll u_{1}} b_{1} \vee \ldots \vee{ }_{b_{n} \ll u_{n}}^{\bigvee} b_{n}
$$

and therefore

$$
u_{1} \vee \ldots \vee u_{n}=\bigvee_{b_{1} \ll u_{1}, \ldots, b_{n} \ll u_{n}} b_{1} \vee \ldots \vee b_{n}
$$

where the right-hand side is a directed join. The conclusion follows by definition of the way-below relation.

The following lemma is an abstract formulation of lemma 1. 
Lemma 6. Let $L$ be a continuous lattice, $b, c$ elements of $L$ and $U, V$ subsets of $L$. Suppose $b \ll c, c \in \mathcal{I}(U)$ (where $\mathcal{I}(U)$ is the ideal generated by $U$ ) and for all $u \in U, \forall d(d \ll u \rightarrow d \in \mathcal{I}(V))$. Then $b \in \mathcal{I}(V)$.

Proof: If $b \ll c$ and $c \leq u_{1} \vee \ldots \vee u_{n}$, where $u_{i} \in U$ for $i=1, \ldots, n$, then $b \ll u_{1} \vee \ldots \vee u_{n}$. By lemma 5 , there exist $b_{1} \ll u_{1}, \ldots b_{n} \ll u_{n}$ such that $b \leq b_{1} \vee \ldots \vee b_{n}$. By the assumption that for all $u \in U, \forall d(d \ll u \rightarrow d \in \mathcal{I}(V))$, we have that $b_{i} \in \mathcal{I}(V)$ for $i=1, \ldots, n$, and therefore $b \in \mathcal{I}(V)$ as well.

Proof of theorem 3: Let $L$ be a continuous lattice. Define relations between elements and subsets of $L$ (or of a base $S$ of $L$ which is a meet-semilattice) in the following way:

$$
\begin{aligned}
a \triangleleft U & \equiv \forall b(b \ll a \rightarrow b \in \mathcal{I}(U)), \\
a \triangleleft_{f} U & \equiv a \in \mathcal{I}(U) .
\end{aligned}
$$

It is easy to verify that $\triangleleft_{f}$ is an infinitary preorder (satisfying in addition dot-left). As for $\triangleleft$ we prove the following:

- Reflexivity holds since $b \ll a$ implies $b \leq a$, so if $a \in U, b \in \mathcal{I}(U)$.

- Transitivity: Suppose $a \triangleleft U$ and $U \triangleleft V$ and let $b \ll a$. By the interpolation property, there exists $c \in L$ such that $b \ll c \ll a$ and therefore $c \in \mathcal{I}(U)$. By lemma $6, b \in \mathcal{I}(V)$, so $a \triangleleft V$. In case we are working with a base $S$ of $L$, the proof requires a closer inspection. By the remark after proposition 8, there exist $c_{1}, \ldots, c_{n}$ in $S$ such that $b \ll \bigvee_{i=1}^{n} c_{i} \ll a$. For all $i \leq n, c_{i} \in \mathcal{I}(U)$, i.e., there exist $u_{i, 1}, \ldots, u_{i, n_{i}}$ in $U$ such that $c \leq u_{i, 1} \vee \ldots \vee u_{i, n_{i}}$ and therefore $b \ll \bigvee_{i \leq n} u_{i, 1} \vee \ldots \vee u_{i, n_{i}}$. By lemma 5 , for all $i \leq n$ and for all $j \leq n_{i}$ there exists $b_{i, j} \ll u_{i, j}$ such that $b \leq \bigvee_{i \leq n} b_{i, 1} \vee \ldots \vee b_{i, n_{i}}$. Since all the $b_{i, j}$ 's are in $\mathcal{I}(V)$, so is $b$.

- Dot - left: If $a \triangleleft U$ and $b \ll a \wedge c$, then $b \ll a$, so $b \in \mathcal{I}(U)$.

If $L$ is distributive, then $\triangleleft_{f}$ also satisfies localization. Localization for $\triangleleft$ is proved as follows: If $a \triangleleft U$ and $b \ll a \wedge c$, then $b \ll a$ and therefore $b \in \mathcal{I}(U)$. Since also $b \leq c$ we have, by distributivity, $b \in \mathcal{I}(U \wedge c)$.

Let $\mathcal{A}$ be the infinitary preorder/formal topology thus defined. We are now going to prove that $\triangleleft_{f}$ is the Stone compactification of $\triangleleft$ :

- $a \triangleleft_{f} U \Rightarrow a \triangleleft U$ since $b \ll a$ implies $b \leq a$, so if $a \in \mathcal{I}(U)$, also $b \in \mathcal{I}(U)$.

- $a \triangleleft_{f} U \Rightarrow\left(\exists U_{0} \subseteq_{\omega} U\right)\left(a \triangleleft_{f} U_{0}\right)$ holds by definition.

- $a \triangleleft U \& U$ finite $\Rightarrow a \triangleleft_{f} U$ : For all $b \ll a, b \leq \bigvee U$ and therefore $a \leq \bigvee U$. Since $U$ is finite, $\bigvee U \in \mathcal{I}(U)$, hence $a \in \mathcal{I}(U)$, i.e., $a \triangleleft_{f} U$.

Finally, the bijection between $L$ and $\operatorname{Sat}(\mathcal{A})$ is given by the two mappings (with $\downarrow a$ replaced by $\downarrow_{S} a$ if $S$ is a base of $L$ )

$$
\begin{array}{rlrl}
\operatorname{Sat}(\mathcal{A}) & \rightarrow L & L & \rightarrow \operatorname{Sat}(\mathcal{A}) \\
U & \mapsto \bigvee U & a & \mapsto \downarrow a
\end{array}
$$


which are seen to be lattice/frame homomorphisms and inverses of each other.

In [HL], a topological representation for distributive continuous lattices is obtained by using the hull-kernel topology on the spectrum of $L$. We recall that the spectrum $\operatorname{Spec}(L)$ of a lattice $L$ is the set of non-top prime elements, i.e. of elements $p$ satisfying

$$
a \wedge b \leq p \text { implies } a \leq p \text { or } b \leq p .
$$

The hull-kernel topology is generated by the subsets $\operatorname{Spec}(L)-\uparrow a$ with $a$ ranging in $L$. For complete lattices this is the same as the extensional topology on the space of completely prime filters on $L, P t(L)$ : Every completely prime filter $\alpha$ on $L$ is of the form $L-\downarrow p$ for a prime $p$ (the supremum of the complement of $\alpha$ in $L$ ). By mapping $p$ in $L-\downarrow p$ an anti-order isomorphism $\phi$ is obtained between $\operatorname{Spec}(L)$ and $\operatorname{Pt}(L)$. Moreover this map induces an isomorphism between the hull-kernel and the extensional topology on these spaces since $\phi(\operatorname{Spec}(L)-\uparrow a)=\{\alpha \in \operatorname{Pt}(L) \mid a \in \alpha\}$. From this observation it follows that the results concerning the spectral theory of (distributive) continuous lattices are obtained in our setting with dualities replaced by equivalences. In particular, corollary 2 is the poinfree part of the result in [HL] stating that distributive continuous lattices are isomorphic to the lattices of opens of sober locally compact topological spaces. This latter result in turn is obtained in our setting from the extensionality of locally Stone formal topologies (cf. proposition 9 below).

A similar representation theorem holds for prime-continuous lattices:

Theorem 4. If $\mathcal{A}$ is a locally Scott infinitary preorder, then $\operatorname{Sat}(\mathcal{A})$ is a primecontinuous lattice.

Proof: As in the proof of theorem 2, we obtain $U=\bigvee\{V \in \operatorname{Sat}(\mathcal{A}): V \lll U\}$ since $b \in i(a)$ implies $\mathcal{A} b \lll \mathcal{A} a$ by definition of locally Scott infinitary preorder.

Theorem 5. Every prime-continuous lattice is isomorphic to the frame of saturated subsets of a locally Scott formal topology.

Proof: Given a prime-continuous lattice $L$, define the following relations between elements and subsets of $L$ (or, as before, of a base $S$ of $L$ which is a meetsemilattice), where $\downarrow U \equiv \bigcup_{u \in u} \downarrow u$ :

$$
\begin{aligned}
a \triangleleft U & \equiv \forall b(b \lll a \rightarrow b \in \downarrow U), \\
a \triangleleft_{s} U & \equiv a \in \downarrow U .
\end{aligned}
$$

The verification that $\triangleleft_{s}$ and $\triangleleft$ are covers is straightforward. Moreover, it is easy to prove that $\triangleleft_{s}$ is the Scott compactification of $\triangleleft$, since we have:

$-a \triangleleft_{s} U \Rightarrow a \triangleleft U$; 
$-a \triangleleft_{s} U \Rightarrow(\exists b \in U)\left(a \triangleleft_{s} b\right)$;

$-a \triangleleft b \Rightarrow a \triangleleft_{s} b$.

The bijection between $L$ and $\operatorname{Sat}(\mathcal{A})$ is obtained as before.

By the representation theorem for continuous lattices via locally Stone infinitary preorders we also obtain an alternative proof of the well known retraction theorem (cf. [Sc]):

Theorem 6. Every continuous lattice is the retract of an algebraic lattice (via a continuous s-r pair).

Proof: Let $L$ be a continuous lattice. By theorem 3, there exists a locally Stone infinitary preorder $\mathcal{A}$ such that $L$ is isomorphic to $\operatorname{Sat}(\mathcal{A})$.

Let $\mathcal{A}_{f}$ be the Stone infinitary preorder defining $\mathcal{A}$. We have the following diagram, where $s$ maps an element $a$ of the base into the subset $i(a)$ and $r$ is the identity map

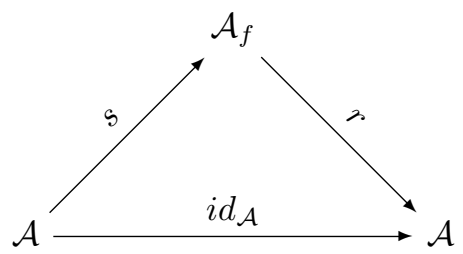

Since $\mathcal{A}$ is locally Stone, by proposition 7 every basic neighbourhood $a$ is equicovered with $i(a)$, and therefore $r$ and $s$ factorize the identity arrow on $\mathcal{A}$ so that the diagram is commutative. By applying the functor Sat we obtain another commutative diagram (in the category of sup-lattices)

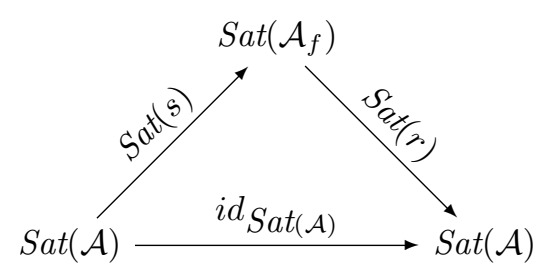

that yields the conclusion since $\operatorname{Sat}\left(\mathcal{A}_{f}\right)$ is an algebraic lattice by proposition 1 .

Then, simply by adding distributivity, and using proposition 2 , we get:

Corollary 3. Every distributive continuous lattice is the retract of a coherent frame (via a continuous s-r pair).

We remark that a different terminology can be found in the literature, where arithmetic is sometimes used instead of coherent when properties of the lattice of compact elements are given in terms of properties of the way-below relation. In view of proposition I.4.7 in [Comp], a coherent lattice is the same as an arithmetic lattice. 


\section{Representation of locally compact spaces}

In this section we will see how locally Stone formal topologies can be identified with sober locally compact topological spaces. The identification takes the form of an equivalence of categories.

As usual when an equivalence has to be proved between pointfree and pointset spaces, the axiom of choice is required in the proof of extensionality (cf. the similar proof for locally compact locales in $[\mathrm{J}]$ ):

Proposition 9. A locally Stone formal topology $\mathcal{A}$ is extensional.

Proof: Suppose that $a$ and $U$ are respectively an element and a subset of the base $S$ of $\mathcal{A}$ and that $a \nless U$. Our claim is that there exists a formal point $\alpha$ of $\mathcal{A}$ such that $a \in \alpha$ and $\alpha \cap U=\emptyset$. From the assumption $a \nless U$ and the fact that $\mathcal{A}$ is a locally Stone formal topology, it follows that there exists $c \in i(a)$ such that $c \nexists_{f} U$. In the continuous lattice $\operatorname{Sat}(\mathcal{A})$ we have $\mathcal{A} c \ll \mathcal{A} a$, so, by the interpolation property, we can inductively define a sequence $D_{0}, D_{1}, \ldots, D_{n}$, $D_{n+1}$ in $\operatorname{Sat}(\mathcal{A})$ such that

$$
\mathcal{A} c \ll D_{n+1} \ll D_{n} \ll \ldots \ll D_{1} \ll D_{0} \ll \mathcal{A} a .
$$

Consider now $F \equiv \bigcup\left\{\uparrow D_{n} \mid n \geq 0\right\}=\bigcup\left\{\uparrow D_{n} \mid n \geq 0\right\}$, where $\uparrow D_{n}=\{U \in$ $\left.\operatorname{Sat}(\mathcal{A}) \mid D_{n} \triangleleft U\right\}$ and $\uparrow D_{n}=\left\{U \in \operatorname{Sat}(\mathcal{A}) \mid D_{n} \ll U\right\}$. Clearly, $F$ is a filter in $\operatorname{Sat}(\mathcal{A})$ and it is Scott open (being a union of basic Scott opens). Moreover, $\mathcal{A} a \in F$ and for all $b \in U, \mathcal{A} b \notin F$. By Zorn's lemma, $F$ extends to a Scott open filter $G$ maximal amongst those containing $\mathcal{A} a$ and having empty intersection with $\{\mathcal{A} b \mid b \in U\}$. By lemma VII.4.3 in $[\mathrm{J}], G$ is prime, and since it is Scott open, it is completely prime. By taking $\alpha \equiv\{b \in S \mid \mathcal{A} b \in G\}$ the desired point is obtained.

We will denote with LSFTop the full subcategory of FTop given by locally Stone formal topologies. By the above proposition, LSFTop is classically a full subcategory of EFTop, the category of extensional formal topologies.

We have:

Proposition 10. If $\mathcal{A}$ is a locally Stone formal topology, the frame of open sets in the extensional topology is a distributive continuous lattice.

Proof: Since $\mathcal{A}$ is extensional by 9 , the frame of opens of $\operatorname{Pt}(\mathcal{A})$ with the extensional topology is isomorphic to $\operatorname{Sat}(\mathcal{A})$. The conclusion follows by corollary 2 .

Proposition 11. If $\mathcal{A}$ is a locally Stone formal topology, the formal space $\operatorname{Pt}(\mathcal{A})$ with the extensional topology is a sober locally compact topological space.

Proof: Let $\alpha$ be a point in $\operatorname{Pt}(\mathcal{A})$, and let $U$ be a neighbourhood of $\alpha$ in the extensional topology. It is not restrictive to suppose $U=\operatorname{ext}(a)$, for some $a$ in the base of $\mathcal{A}$. Since $a \triangleleft i(a)$ and $a \in \alpha$, by definition of formal points there exists $c \in i(a)$ such that $c \in \alpha$. In $\operatorname{Sat}(\mathcal{A})$ we have $\mathcal{A} c \ll \mathcal{A} a$. Let $F$ be the $\operatorname{Scott}$ 
open filter constructed as in the proof of proposition 9. We claim that $\operatorname{ext}(F)$ is a compact neighbourhood of $\alpha$ contained in $\operatorname{ext}(a)$.

It is clear that $\operatorname{ext}(F)$ is a neighbourhood of $\alpha$ contained in $\operatorname{ext}(a)$ since $c \triangleleft F$ implies $\operatorname{ext}(c) \subseteq \operatorname{ext}(F)$ and $F \triangleleft a$ implies $\operatorname{ext}(F) \subseteq \operatorname{ext}(a)$. As for compactness, suppose that $\operatorname{ext}(F) \subseteq \bigcup_{i \in I} \operatorname{ext}\left(U_{i}\right)$. By extensionality we have $F \triangleleft \bigcup_{i \in I} U_{i}$, and in the frame $\operatorname{Sat}(\mathcal{A})$,

$$
F \leq \bigvee_{U_{0} \subseteq \omega} U_{i \in I} U_{0}
$$

where the right-hand side is a directed join. Since $F$ is a Scott open filter, there exists $U_{0} \subseteq_{\omega} \bigcup_{i \in I} U_{i}$ such that $F \leq U_{0}$. Therefore, there exists $I_{0} \subseteq_{\omega} I$ such that $F \triangleleft \bigcup_{i \in I_{0}} U_{i}$, that is, such that $\operatorname{ext}(F) \subseteq \bigcup_{i \in I_{0}} \operatorname{ext}\left(U_{i}\right)$.

Conversely, we have the following:

Proposition 12. Let $\langle X, \Omega(X)\rangle$ be a sober locally compact topological space. Then there exists a locally Stone formal topology $\mathcal{A}$ such that $\langle X, \Omega(X)\rangle$ is isomorphic to the formal space $\operatorname{Pt}(\mathcal{A})$ with the extensional topology.

Proof: We already know from the equivalence between formal topologies and sober topological spaces that the basic monoid of the formal topology corresponding to the topological space $X$ consists of the basic opens with monoid operation given by set-theoretic intersection; the cover is defined by

$$
a \triangleleft U \equiv a \subseteq \bigcup U
$$

where $a$ is a basic open of $X, U$ is a collection of basic opens, and $\subseteq$ is the usual set-theoretic inclusion. It is straightforward, by the validity of the rules for formal topology in their extensional reading, that this defines a formal topology. In order to prove that this is indeed a locally Stone formal topology, we observe that by local compactness of the space $X$, the cover can be equivalently defined as follows:

$$
a \triangleleft U \equiv(\forall b \ll a)\left(b \triangleleft_{f} U\right)
$$

with $b \triangleleft_{f} U$ iff there exists a finite subset $U_{0}$ of $U$ such that $b \subseteq \bigcup U_{0}$.

For one direction one just uses that $b \ll a$ iff there exists a compact open $k$ with $b \subseteq k \subseteq a$. The converse follows since for any (basic) open set $a$ in a locally compact topological space, $a=\bigcup_{b \ll a} b$.

Let $F$ be the functor from the category FTop to the category Top of topological spaces mapping a formal topology $\mathcal{A}$ to the formal space $\operatorname{Pt}(\mathcal{A})$ with the extensional topology. It is well known that $F$ gives an equivalence between the category EFTop of extensional formal topologies and the category STop of sober topological spaces. By a characterization of equivalence of categories (see [MCL]), F is full, faithful and dense (that is, it is bijective on morphisms and surjective, up to isomorphism, on objects). By proposition 11, $F$ restricts to a functor, that we will denote by $\bar{F}$ between the category LSFTop and the category SLCTop of sober locally compact topological spaces. Since LSFTop and SLCTop are full subcategories of EFTop and STop, respectively, $\bar{F}$ is 
full and faithful. By proposition $12, \bar{F}$ is dense. Therefore, by restriction of the equivalence between extensional formal topologies and sober topological spaces we have:

Theorem 7. The category of locally Stone formal topologies is equivalent to the category of sober locally compact topological spaces.

We observe that we can obtain the equivalence stated above in an alternative indirect way. First, by using the functor Sat that to a formal topology associates its frame of saturated subsets, by theorem 2 and theorem 3 we obtain an equivalence between the category of locally Stone formal topologies and the category of distributive continuous lattices. By composing this equivalence with the equivalence between the category of distributive continuous lattices and the category of sober locally compact topological spaces (see e.g. thm. 7.2.16 in [AJ]), we get an equivalence between the category of locally Stone formal topologies and the category of sober locally compact topological spaces.

\section{Concluding remarks and related work}

The correspondence between formal spaces and locally compact frames or equivalently, distributive continuous lattices, has been studied in detail in [Si] using neighbourhood systems and generators for cover relations.

An important difference with her work is that here we work constructively, and in addition extend the representation to nondistributive structures using the generalization of the notion of cover relation to that of infinitary preorder.

Another related work is [SiS] where two representations for regular locally compact spaces, one based on domains, another on formal spaces, are compared.

Representations of Scott domains based on formal spaces are given in [Si1,SVV]. In the latter work it is proved that any Scott domain is isomorphic to the partially ordered structure given by the formal points of a Scott formal topology, and an essential use of the positivity predicate is made. Here instead we represent continuous lattices via formal opens of suitable formal topologies.

A problem left open here is the extension of such a representation to lattices that are complete only with respect to directed joins, namely continuous dcpo's, that generalize both continuous lattices and Scott domains. Lattices of this kind are used in the domain-theoretic approach to integration (cf. [E,EN,EN1]).

\section{Acknowledgement}

This research partly originates from my work in the EPSRC project "Foundational Structures in Computer Science" at Imperial College, Department of Computing, that gave me the opportunity to become acquainted with the literature on continuous lattices. 


\section{References}

[AJ] S. Abramsky, A. Jung. Domain theory, in "Handbook of Logic in Computer Science", vol. 3, Clarendon Press, Oxford, pp. 1-168, 1994.

[A] P. Aczel. An introduction to inductive definitions, in "Handbook of Mathematical Logic", J. Barwise (ed), North-Holland, pp. 739-782, 1977.

[BH] B. Banaschewski, R.-E. Hoffmann (eds), "Continuous Lattices", Lecture Notes in Mathematics 871, pp. 209-248, Springer, 1981.

[BS] G. Battilotti, G. Sambin. A uniform presentation of sup-lattices, quantales and frames by means of infinitary preordered sets, pretopologies and formal topologies, Preprint no. 19, Dept. of Pure and Applied Mathematics, University of Padova, 1993.

[JC] J. Cederquist. A machine assisted formalization of pointfree topology in type theory, Chalmers University of Technology and University of Göteborg, Sweden, Licentiate Thesis, 1994.

[JC1] J. Cederquist. An implementation of the Heine-Borel covering theorem in type theory, this volume.

[JC2] J. Cederquist. A machine assisted proof of the Hahn-Banach theorem, Chalmers University of Technology and University of Göteborg, 1997.

[CCN] J. Cederquist, T. Coquand, S. Negri. The Hahn-Banach theorem in type theory, to appear in "Twenty-Five Years of Constructive Type Theory" G. Sambin and J. Smith (eds), Oxford University Press.

[CN] J. Cederquist, S. Negri. A constructive proof of the Heine-Borel covering theorem for formal reals, in "Types for Proofs and Programs", S. Berardi and M. Coppo (eds), Lecture Notes in Computer Science 1158, pp. 62-75, Springer, 1996.

[E] A. Edalat. Domain theory and integration, Theoretical Computer Science 151, pp. 163-193, 1995.

[EN] A. Edalat, S. Negri. The generalized Riemann integral on locally compact spaces (extended abstract), in "Advances in Theory and Formal Methods of Computing", A. Edalat, S. Jourdan and G. McCusker (eds), World Scientific, Singapore, 1996.

[EN1] A. Edalat, S. Negri. The generalized Riemann integral on locally compact spaces, Topology and its Applications (in press).

[FG] M. P. Fourman, R.J. Grayson. Formal spaces, in "The L. E. J. Brouwer Centenary Symposium", A. S. Troelstra and D. van Dalen (eds), pp. 107122, North-Holland, Amsterdam, 1982.

[Comp] G. Gierz, K.H. Hoffmann, K. Keimel, J. D. Lawson, M. Mislove, D. S. Scott. "A Compendium on Continuous Lattices", Springer, 1980.

[HL] K.H. Hoffmann, J.D. Lawson. The spectral theory of distributive continuous lattices, Transactions of the American Mathematical Society 246, pp. 285310, 1978.

[HM] K.H. Hoffmann, M.W. Mislove. Local compactness and continuous lattices, in "Continuous Lattices", B. Banaschewski and R.-E. Hoffmann (eds), op. cit..

[I] J.R. Isbell. Atomless parts of spaces, Mathematica Scandinavica 31, pp. 5-32, 1972.

[J] P. T. Johnstone. "Stone Spaces", Cambridge University Press, 1982.

[JT] A. Joyal, M. Tierney. An extension of the Galois theory of Grothendieck, Memoirs of the American Mathematical Society 51, no. 309, pp. 1-71, 1984. 
[MCL] S. MacLane. "Categories for the Working Mathematician", Springer, 1971.

[ML] P. Martin-Löf. "Notes on Constructive Mathematics", Almqvist \& Wiksell, Stockholm, 1970.

[ML1] P. Martin-Löf. "Intuitionistic Type Theory", Bibliopolis, Napoli, 1984.

[MP] C.J. Mulvey, J.W. Pelletier. A globalization of the Hahn-Banach theorem, Advances in Mathematics 89, pp. 1-60, 1991.

[N] S. Negri. Stone bases, alias the constructive content of Stone representation, in "Logic and Algebra", A. Ursini and P. Aglianó (eds), Dekker, New York, pp. 617-636, 1996.

[N1] S. Negri. "Dalla topologia formale all'analisi", Ph. D. thesis, University of Padova, 1996.

[NS] S. Negri, D. Soravia. The continuum as a formal space, Archive for Mathematical Logic (in press).

[NV] S. Negri, S. Valentini. Tychonoff's theorem in the framework of formal topologies, The Journal of Symbolic Logic (in press).

[NPS] B. Nordström, K. Petersson, J. Smith, "Programming in Martin-Löf's Type Theory", Oxford University Press, 1990.

[S] G. Sambin. Intuitionistic formal spaces - a first communication, in "Mathematical Logic and its Applications", D. Skordev (ed), Plenum Press, New York, pp. 187-204, 1987.

[S1] G. Sambin. Intuitionistic formal spaces and their neighbourhood, in "Logic Colloquium '88", R. Ferro et al., (eds), pp. 261-285, North-Holland, Amsterdam, 1989.

[SVV] G. Sambin, S. Valentini, P. Virgili. Constructive domain theory as a branch of intuitionistic pointfree topology, Theoretical Computer Science 159, pp. 319-341, 1996.

[Sc] D.S. Scott. Continuous lattices, in "Toposes, Algebraic Geometry and Logic", F.W. Lawvere (ed), Lecture Notes in Mathematics 274, pp. 97136, Springer, 1972.

[Sc1] D.S. Scott. Models for various type-free calculi, in "Logic, Methodology and Philosophy of Science IV", P. Suppes et al. (eds), North-Holland, pp. 157-187, 1973.

[Si] I. Sigstam. "On formal spaces and their effective presentations", Ph. D. thesis, Report 1990:7, Department of Mathematics, University of Uppsala.

[Si1] I. Sigstam. Formal spaces and their effective presentation, Archive for Mathematical Logic 34, pp. 211-246, 1995.

[SiS] I. Sigstam, V. Stoltenberg-Hansen. Representability of locally compact spaces by domains and formal spaces, Theoretical Computer Science 179, pp. 319-331, 1997.

[SLG] V. Stoltenberg-Hansen, I. Lindström, E.R. Griffor. "Mathematical Theory of Domains", Cambridge University Press, 1994.

[V] S. Vickers. "Topology via Logic", Cambridge University Press, 1989. 